\title{
Action dual-tasks reveal differential effects of visual imagery perspectives on motor performance
}

Callow, Nichola; Edwards, Martin Gareth; Jones, Alex Lee; Hardy, Lew; Connell, Stephanie

\section{Quarterly Journal of Experimental Psychology}

DOI:

$10.1177 / 1747021818811464$

Published: 01/06/2019

Peer reviewed version

Cyswllt i'r cyhoeddiad / Link to publication

Dyfyniad o'r fersiwn a gyhoeddwyd / Citation for published version (APA):

Callow, N., Edwards, M. G., Jones, A. L., Hardy, L., \& Connell, S. (2019). Action dual-tasks reveal differential effects of visual imagery perspectives on motor performance. Quarterly Journal of Experimental Psychology, 72(6), 1401-1411.

https://doi.org/10.1177/1747021818811464

\footnotetext{
Hawliau Cyffredinol / General rights

Copyright and moral rights for the publications made accessible in the public portal are retained by the authors and/or other copyright owners and it is a condition of accessing publications that users recognise and abide by the legal requirements associated with these rights.

- Users may download and print one copy of any publication from the public portal for the purpose of private study or research.

- You may not further distribute the material or use it for any profit-making activity or commercial gain

- You may freely distribute the URL identifying the publication in the public portal ?
}

Take down policy

If you believe that this document breaches copyright please contact us providing details, and we will remove access to the work immediately and investigate your claim. 
Title: Action dual-tasks reveal differential effects of visual imagery perspectives on motor performance

Callow, N. ${ }^{1}$, Edwards, M.G. ${ }^{2}$, Jones, A.L. ${ }^{3}$, Hardy, L. ${ }^{2}$, \& Connell, S. ${ }^{2}$

Accepted in current form for Quarterly Journal of Experimental Psychology

$8^{\text {th }}$ July 2018

${ }^{1}$ Institute for the Psychology of Elite Performance, School of Sport, Health and Exercise Sciences, Bangor University, UK

${ }^{2}$ Institut de recherchesen sciences psychologiques, Université catholique de Louvain, Belgium

${ }^{3}$ Department of Psychology, Swansea University, UK 


\begin{abstract}
Imagery research has identified two main visual perspectives, External Visual Imagery (EVI, third-person) and Internal Visual Imagery (IVI, first-person). Based upon findings from brain imaging literature showing different neural substrates are recruited for IVI and EVI perspectives, and that IVI activates motor system brain areas, we hypothesized that a concurrent action dual-task would cause greater interference in performance for IVI than EVI. In a first experiment, participants were allocated to either an IVI or an EVI group, and were tasked with moving an onscreen marker towards a target in three blocked conditions; imagery, imagery with a concurrent motor dual-task of sequencing, and a math control. An interaction between imagery group and condition was driven by greater Root Mean Square Error for participants in the dual-task condition in the IVI group compared to the EVI group. We replicated the experiment with an eye tracking objective measure of IVI, the results again showed that participants in the IVI group made more errors in motor movements, and an interference effect in eye movements, during the dual-task sequencing condition compared to the EVI group. The results of the two experiments reveal that a secondary motor task does interfere with IVI, providing behavioural evidence that IVI appears to rely on motor system processes more than EVI. These results have important implications for the use of visual imagery perspectives across a number of domains, with the paper being an essential reference for those conducting visual imagery perspectives research.
\end{abstract}


In the cognitive neuroscience literature, visual imagery is delineated into the firstperson perspective where the imaginer looks out through his or her own eyes, or the thirdperson perspective where an observational view is taken of one's own or other's actions (e.g., Ganesh, van Schie, Cross, de Lange, \& Wigboldus, 2015, Vogeley \& Fink, 2003). It is established that visual imagery is central to a variety of high level cognitive and motor functions (e.g., Kosslyn \& Shwartz, 1977; Marks, 1973), with its systemic use shown to facilitate learning and performance (see Carrasco \& Cantalapiedra, 2016; Driskell, Copper, \& Moran 1994 for reviews). Moreover, recent research demonstrates that the perspective used by an individual differentially influences cognitive, emotional and behavioural responses (e.g., Libby, Valenti, Hines, \& Eibach, 2014). These differential responses allude to the possibility that different cognitive and neural processes underpin perspectives. The present studies explores the cognitive processes underlying imagery perspectives using an action dual-task paradigm where we assume that performing an action will use motor neural processes, and this activity will be disruptive to any imagery cognitive processes using the same neural processes. Given the research related to imagery perspectives in cognitive neuroscience and sport psychology, we purposefully draw across both disciplines in order to provide additive power to aid our understanding of visual imagery perspectives and cognitive functioning (cf. Beilock \& Gonso, 2008).

In the sport psychology research literature, visual imagery perspective is commonly referred to as internal visual imagery (IVI) and external visual imagery (EVI). IVI refers to a first-person perspective, and is where the imaginer looks out through his or her own eyes while performing the action. EVI refers to a third-person perspective, and is where the imaginer watches him or herself performing the action from an observer's position; as if watching him or herself on television (Callow \& Roberts, 2013; Hardy, 1997). The use of IVI and EVI has been shown to have different impacts on motor performance, moderating the 
efficacy to perform certain tasks. For example, in a series of three studies employing slalom line-based motor performance activities, where changes in movement in relation to precise spatial and temporal locations were required (i.e., driving simulator, down-hill slalomrunning, ski-slalom), the use of IVI produced more accurate motor performance than EVI (Callow, Roberts, Hardy, Jiang, \& Edwards, 2013). Conversely, in a series of three studies using tasks relying heavily upon the use of form for their successful motor performance (i.e., karate-kata, gymnastics floor routine, bouldering), the use of EVI was found to have a superior influence on performance compared to the use of IVI. Furthermore, the feeling of a movement, known as kinaesthetic imagery, has demonstrated additive performance effects beyond that of visual imagery, producing significant performance gains over and above EVI for form-based tasks (Hardy \& Callow, 1999), and over and above IVI for slalom-based tasks (Callow, Jiang, Roberts, \& Edwards, 2017). Behaviourally, these results provide evidence for the distinctiveness of the visual and kinaesthetic modalities. A cognitive explanation for these effects has been proposed where the use of imagery can benefit motor performance by allowing individuals to supplement information that is already available from the physical movement (Hardy, 1997).

More recently, neurocognitive approaches have investigated plastic changes in the human motor action system resulting from imagery (see Di Rienzo et al., 2016 for a review), with overarching evidence that imagery produces functional changes in brain activation that may facilitate motor performance (Frank \& Schack, 2017). For example, using transcranial magnetic stimulation, Pascual-Leone et al. (1995) investigated plastic changes in the human motor action system resulting from physical practice and imagery of a key pressing task. Although physical practice was superior to imagery in terms of performance, both physical and imagery practice led to the same plastic changes, namely an equally increased size of the cortical representation for the control of finger muscle groups that were used in the task. 
More recently, using functional magnetic resonance imaging, Zhang et al. (2014) reported changes in cognitive and sensory resting state networks in various brain systems after the learning of a sequential finger tapping task using imagery, while no changes in connectivity were found in the control condition (i.e., no practice).

Based on this body of literature, it is intuitively appealing to hypothesize that differential behavioural responses for visual imagery perspectives are likely caused by differential underlying neural processes. However, due to conceptual confounds in the literature, it is currently difficult to establish if there is a dissociation of the neural areas used for the different visual imagery perspectives. For example, studies applying internal imagery can confound external visual and kinaesthetic modalities (e.g., Jeannerod, 1994, Ruby \& Decety, 2001), and some studies using external imagery have someone else, rather than imagery of oneself, as the agent (e.g., Fourkas, Ionta, \& Aglioti, 2006; Ruby \& Decety, 2001), which can produce different behavioural performance outcomes (Callow \& Hardy, 2004). While several fMRI studies (e.g., Guillot, Collet, Nguyen, Malouin, Richards, \& Doyon, 2009) are clear to make distinctions between imagery modalities (i.e., visual and kinaesthetic), these studies again have not examined visual perspective differences. That said, in a recent study, Jiang, Edwards, Mullen, and Callow (2015) addressed some of these limitations. Specifically, participants with high imagery ability were asked to imagine a movement (running up the stairs) from both IVI and EVI while in an fMRI scanner. Results revealed neural activation of the supplementary motor area, and more generally BA6 for both conditions, a finding consistent with other studies examining the neural underpinnings of movement visual imagery (Ross, Tkach, Ruggieri, Lieber, \& Lapresto, 2003). Interestingly, contrast analyses showed neural differences in activation when performing the different visual imagery perspectives. The use of IVI activated occipital, parietal and frontal brain areas (i.e., areas associated with the dorsal stream; Norman, 2002). The use of EVI activated 
similar areas to a much lesser extent, and EVI also resulted in activation of occipital ventral stream areas, similarly to neuroimaging studies examining 3PP taking (Ruby \& Decety, 2001). This sole evidence supports a dissociation of the neural areas used for the different visual imagery perspectives.

Despite a lack of neural evidence, behavioural evidence suggests that IVI and EVI may be based on different cognitive (neural) processes. In the literature, it is suggested that first-person perspectives (such as IVI) are thought to utilize an egocentric reference frame (i.e., the representation of object locations in relation to the individual and their physical configuration, as in a polar coordinate system) and dorsal stream cognitive processes (Norman, 2002), with projections from occipital and parietal to motor and frontal. Conversely, third-person perspectives (such as EVI) are considered to use an allocentric reference frame (i.e., an object semantic framework that is independent from the individual, as in a Cartesian coordinate system), and use ventral stream cognitive processes (Norman, 2002), with weaker connections to the motor cortex (Ungerleider \& Mishkin, 1982). Interestingly, Deyzac, Logie, and Denis (2006) used a spatial interference dual-task paradigm to investigate the cognitive components of working memory (imagery) involved in processing spatial descriptions. Participants were asked to draw maps of a spatial environment from memory based on instructions from a route or survey perspective. The route perspective required participants to imagine moving to new points using an egocentric frame of reference (i.e., first-person perspective / IVI) before creating their maps.

Conversely, the survey perspective required participants to imagine the environment from an allocentric, fixed bird's eye view (i.e., third-person perspective / EVI). While accuracy did not differ between perspectives at baseline, the addition of the spatial interference task significantly impaired accuracy for the route perspective (i.e., first-person perspective / IVI). The survey perspective was unaffected by spatial interference (i.e., third-person perspective / 
EVI). These results suggested that the route perspective relied on the same cognitive processes as those used by the spatial interference task, and furthermore, that the survey perspective used independent cognitive processes. As spatial cognition is thought to use dorsal stream neural processes (e.g., Ungerleider \& Mishkin, 1982), it is possible to infer that these data suggest that first-person perspective / IVI relies on dorsal stream cognitive processes, whereas third-person perspective / EVI does not rely on dorsal stream cognitive processes.

In the present paper, we sought to extend the research of Deyzac et al. (2006), and test whether a motor dual-task could demonstrate a cognitive dissociation between IVI and EVI processes using a motor performance behavioural task. In Experiment 1, we used a computerized performance task where participants were asked to move a marker toward a target on screen while performing a secondary motor task, as well as using either EVI or IVI in between trials to prime performance. Firstly, based on previous literature, we hypothesized that both perspectives of imagery (IVI and EVI) would significantly improve performance relative to a non-imagery control condition. Then, for the dual-task, we hypothesize that if IVI involves dorsal stream cognitive processes, the motor dual-task (which we assume will use dorsal stream cognitive processes), should interfere with the performance benefits gained from the use of IVI. However, EVI, which we assume activates areas in the ventral stream (and not the dorsal stream) should show no changes in the EVI performance priming for the dual-task.

In Experiment 2, we aimed to replicate Experiment 1 (strengthening our results), but in addition incorporated eye tracking to provide an objective measure of imagery use during the experimental tasks. We also aimed to investigate whether eye movements, physical correlates of visual imagery (cf., Johansson, Holsanova, \& Holmqvist, 2011; Poiroux, et al., 2015), differed for the visual imagery perspectives. The results from both experiments 
provide important information regarding the cognitive (neural) processes underpinning visual imagery perspectives that drive differential behavioural performance. These results will have relevance not only to methodological considerations across disciplines, but they will also provide valuable new knowledge that can be applied to allied domains of rehabilitation and sport.

\section{Experiment 1}

\section{Method}

Participants and design. Forty-two university undergraduates (Age $M=25.30, S D=$ 3.90, 24 males) were screened for imagery ability using the Vividness of Movement Imagery Questionnaire-2 (VMIQ-2; Roberts, Callow, Hardy, Markland \& Bringer, 2008). Across the three imagery subscales (IVI, EVI and kinaesthetic, all participants scored above the cut-off criteria of 36 (cf. Callow et al., 2013) indicting least moderate imagery ability, with an average of $M=24.07, S D=6.88$. All participants were right handed, as assessed by the Edinburgh Handedness Inventory (EHI; Dragovic, 2004), and had normal or corrected to normal vision.

We used a mixed Group (IVI, EVI) and Condition (Imagery, Dual-Task with Imagery, Math Control) design, with repeated measures on Condition. As preference and ability of visual imagery perspective are correlated (Callow \& Roberts, 2010) participants were divided and assigned to the IVI or EVI group based on their VMIQ-2 visual imagery preference scores. Specifically, those who scored 4 and above indicating a preference for IVI were allocated to the IVI group, and those who scored 6 and above indicating a preference for EVI were allocated to the EVI group. Where a participant indicated no preference, they were randomly assigned to a group.

Equipment, tasks and procedure. Prior to experimentation, we asked participants to complete the EHI and the VMIQ-2. The seven items of the EHI measure handedness in terms 
of left, right, or ambidextrous by examining preference in a number of different activities (e.g., writing). Participants with a Laterality Quotient of 40 or greater, indicating predominantly right-handedness were included in the study. All 42 participants included in the study were right-handed.

The VMIQ-2 contains 12 items that measure imagery ability on a Likert scale from 1 (perfectly clear image) to 5 (no image at all) for IVI, EVI and kinaesthetic imagery (KIN) separately. Participants were asked to image with their eyes open when completing the questionnaire. There exists inter-individual variance in imagery ability, which can impact on the effectiveness of imagery interventions (Roberts et al., 2008). Therefore, in the present study, only participants with moderate to good imagery ability (a score of 36 or below) were included (e.g., see Callow et al., 2013 for similar inclusion criteria). The VMIQ-2 also measures an individual's preferred imagery perspective on an 11-point Likert scale ranging from 0 (strong preference for internal visual imagery) to 5 (no preference) to 10 (strong preference for external visual imagery) their visual imagery preference. Imagery perspective preference is an important moderator of imagery ability (Hall, 1997). As higher imagery ability is correlated with effectiveness of interventions (Isaac, 1992), Callow and Hardy (2004) proposed that imagery preference should be controlled for when examining imagery effects. As previous highlighted, in order to control for preference, we allocated participants to the visual perspective group that matched with their preference.

For the main experiment, we asked participants to sit facing a Mitsubishi XC-3730C 32" CRT monitor ( $800 \times 600$ pixels), with their eye level at the centre of the monitor, and to make responses in the primary task using a 3400 DrawingBoard III data tablet (sampling $200 \mathrm{hz}$, precision $0.125 \mathrm{~mm}$ ). For the dual-task sequencing condition, performance on the secondary task (sequence finger tapping) was recorded using custom software, written in $\mathrm{C}++$ on a Pentium $450 \mathrm{MHz}$ system, collected using a keyboard located to the right hand side 
of the participant. Participants were required to wear PLATO goggles (Portable LiquidCrystal Apparatus for Tachitoscopic Occlusion; Translucent Technologies Inc.). The use of goggles enabled participants to image with their eyes open while removing any environmental distracters and preventing any differences in cortical activation which may result from eyes being open in movement task and then closed in imagery (Holmes, 2007). See Figure 1 for equipment set-up.

The primary task was an aiming task. The task started with one practice trial (excluded from data analyses), and 15 experimental trials for each of the three conditions (Imagery, Dual-Task Sequencing with Imagery, or Math Control). The two groups of Imagery allowed for participants to perform IVI or EVI perspective imagery dependent on group assignment. To perform the aiming task, participants picked up a digital pen with their right hand, and they were asked to place a cursor line (10 x $2 \mathrm{~mm}$ in size $)$ on a start line presented centrally at $30 \mathrm{cms}$ from the left side of the computer screen. The digital pen was on the tablet underneath the monitor, and the pen aligned with the position of the cursor line on the monitor. A tone signalled the onset of a target line (15 x $2 \mathrm{~mm}$ in size $)$ on the right side of the screen, and participants were required to move the digital pen, and hence the cursor line, across the digital tablet from the start to the target line as quickly as they could, in a single fluid movement. There were three possible targets, which differed in their horizontal distance from the start line $(460 \mathrm{~mm}, 530 \mathrm{~mm}$, and $600 \mathrm{~mm})$. These distances were derived from pilot testing, with the largest distance $(600 \mathrm{~mm})$ represented the maximum distance within comfortable reach without any torso movement for the majority of the population. See Figure 2 for a diagrammatical representation of the task. The lesser distances were as evenly spaced while remaining distinct. Range and order effects were minimized by randomizing the target distances within each condition and counterbalancing the conditions across participants (Grice, 1968; Poulton, 1973). The starter line was only visible until the participant had 
initiated movement, with the target line remaining visible. Further, at the start of each trial, the PLATO goggles that participants were wearing changed from opaque to clear lenses (taking 1ms), allowing them to see and perform that task. At the end of each trial, the lenses turned back to opaque (taking approximately $3-5 \mathrm{~ms}$ ), and remained opaque until the secondary task was completed and the trial ended. The experiment was carried out in a darkened environment to prevent contextual cues facilitating accuracy of movement (Krigolson \& Heath, 2004). Participants received 2000ms to initiate and complete their movements before the trial was rendered null. Participants were asked to complete the task in a single, swift movement, keeping their body still, and using their eyes and arm to move during the task. They were also told that their accuracy and reaction time would be monitored.

Both imagery groups in the imagery conditions were asked to image successfully performing the task, with participants in the IVI group asked to image "through their own eyes" and participants in the EVI group asked to image as if they "were watching themselves on television". Recent work has suggested that the switch from an egocentric (first-person perspective / IVI) to allocentric (third-person perspective / EVI) view occurs at $135^{\circ}$ (Burgess, 2006; Waller \& Hodgson, 2006). Further, research highlights that EVI is implemented from a range of angles (Callow \& Roberts, 2010). In order to ensure an EVI view, we asked participants to use an EVI angle of $140^{\circ}$ from the target, demonstrated to the participants using a line drawing of the experimental task scene. In both perspective groups, it was stressed that during imagery, the whole movement of the task should be incorporated, and not just the endpoint (i.e., participants should imagine moving the marker to the target fully). Participants were instructed to image only from their assigned visual perspective. The experimenter also described kinaesthetic imagery in order to ensure that participants could distinguish it from visual imagery perspectives, and to highlight that kinaesthetic imagery 
must not be used. To reduce variance in imagery further, participants read an imagery script detailing the task from the perspective IVI or EVI (depending upon the group), ensuring that all of the participants performed the same imagery.

For the dual-task, participants were asked to imagine successfully performing the task (as described in the paragraph above) while performing a physical secondary motor task. This task required participants to enter a sequence using their right hand into a computer keyboard, by pressing four keys in a specific order using all four fingers (F-G-H-J-J-H-G-F) before pressing the space bar to signal completion. Participants were permitted to look at the location of the keys before starting the trial, and each of the required keys was tactually available via the use of fine sandpaper overlaid on the keys. The dual-task was selected as finger sequence execution activates the primary sensorimotor cortex, supplementary motor area and premotor cortex (Witt, Laird, \& Meyerand, 2008), and sequence processing leads to activation of the posterior parietal areas (Catalan, Honda, Weeks, Cohen, \& Hallett, 1998). Therefore, finger sequencing fully activates the dorsal stream, allowing us the potential to interfere the cognitive processes involved in IVI and EVI.

The imagery, and imagery with dual-task sequencing, conditions were compared to a control condition of maths. Participants completed one mental arithmetic question (e.g., $(16+$ 4) +3), preventing the participant from use of any imagery. These maths questions were identical for all participants, but with a randomised order. Participants announced their answers verbally.

After each of the three different conditions, participants completed relevant sections of a post-experimental questionnaire, and then given detailed instructions of the following experimental condition. After all conditions, participants then completed the remainder of the post-experimental questionnaire. Participants did not receive any feedback during the experiment. The post-experimental questionnaire evaluated imagery use and adherence to 
assigned perspective; whether they switched from their assigned perspective, and whether they experienced kinaesthetic imagery. Participants indicated their responses on a ten-point Likert scale, with lower scores indicating greater adherence, and less switching and kinaesthetic imagery experience. Participants who showed compromised adherence in imagery perspective or modality were removed from analyses.

Data analyses. The dependent measure was of the aiming task was root mean square error (RMSE: Krigolson, Gyn, Tremblay, \& Heath, 2006; Proteau, Tremblay, \& Dejaeger, 1998). RMSE measures the average magnitude of error by squaring the sum of squared residuals and taking the square root of the average of these errors. Thus, by taking the final marker position and calculating distance along the $\mathrm{x}$-axis from the target, we calculated RMSE (in millimetres) for each condition. The data were analysed using a 2 (Group; between) x 3 (Condition; within) ANOVA.

\section{Results}

Examination of the post experimental questionnaires revealed that two participants failed to adhere to instructions and experienced kinaesthetic imagery. Data from the remaining 40 participants (Age $M=25.27, S D=3.87,22$ males), with 20 in each perspective group, were used in the subsequent analysis. All participants adhered to their assigned perspective (Imagery $M=1.00, S D=0.00$; Sequencing with Imagery $M=1.05, S D=0.22$ ), and did not switch from their allocated perspective in either condition (Imagery $M=2.48, S D$ $=1.52$; Sequencing with Imagery $M=2.79, S D=1.49$ ). As imagery ability has been shown to be positively correlated with imagery intervention effectiveness (Hall, Buckolz, \& Fishburne, 1989), we checked whether there were differences between groups in imagery ability. An independent samples t-test demonstrated no significant differences in imagery ability between IVI and EVI groups, $t(38)=0.89, p=.37, \beta=.14$, indicating the screening procedure and assignment worked as intended. 
The ANOVA of performance RMSE showed no main effect for group, $F(1,38)=$ $0.04, p=.83, \beta=.05$, but a significant main effect for condition, $F(1,38)=11.59, p<.001$, $\eta^{2}=.23, \beta=.99$. This showed that Imagery $(M=72.11 \mathrm{~mm})$ resulted in lower errors than Imagery with Sequencing, $(M=87.44 \mathrm{~mm})$, with both having lower errors than the Math Control condition $(M=103.47 \mathrm{~mm})$. The differences between conditions were qualified by a significant interaction between imagery group and condition, $F(1,38)=11.13, p<.001, \eta^{2}=$ $.23, \beta=.99$ (see Figure 3). Post-hoc one-way ANOVAs for each experimental condition showed that the interaction was driven by a significantly greater RMSE in the IVI compared to EVI group for the Sequencing with Imagery condition, $F(1,39)=7.81, p=.008, \eta^{2}=.17$, $\beta=.71$. There were no differences between IVI and EVI groups in the Imagery or Math Control conditions, both $F_{\mathrm{s}}<1.05$. This finding demonstrates that the motor interference task resulted in the deterioration of performance only when using IVI.

\section{Discussion}

The results provided several findings regarding the use of imagery. First, the use of a visual imagery perspective reduced the average error made by participants in the task, as evidenced by the significant main effect of condition, thus supporting the wealth of research demonstrating the beneficial effects of imagery on learning and human performance (Carrasco \& Cantalapiedra, 2016; Driskell, et al., 1994). Secondly, for those participants using EVI, performance was unaffected by the introduction of a sequence dual-task. However, for those in the IVI group, the sequence dual-task resulted in poorer performance. These findings support the notion that perspective produces differential responses (e.g., Libby, et al., 2014) and complement the findings of Deyzac, et al. (2006) who reported dualtask interference of first-person imagery with a concomitant sequencing task, while thirdperson imagery remained unaffected. This suggests that IVI may be considered related to first-person imagery, and furthermore, that IVI involves dorsal stream visuo-motor neural 
processes, supporting our original hypotheses. In a second experiment, we aimed to replicate and extend these findings.

One criticism of imagery research concerns the subjective nature of its measurement (Levine, Warach, \& Farah, 1985). Self-reports of imagery ability have been shown to have poor correlation with more objective questionnaires (Moreau, Clerc, Mansy-Dannay, \& Guerrien, 2010), and simple demand characteristics can lead participants to overstate their imagery ability (for example, in the VMIQ-2; Allbutt, Ling, Rowley, \& Shafiullah, 2011). One objective measure of imagery use using a behavioural marker is the measurement of eye movements. To elaborate Hebb (1968) argued that eye movements evoked when viewing an action were identical to those when imaging the same action, indeed more recent research demonstrates elements of congruence in eye movements between action execution, observation and imagery (McCormick, Causer, \& Holmes, 2013). It is proposed that this congruence occurs because eye movements are indicative of the cognitive attention processes underlying tasks such as reading or visual search, (Liversedge \& Findlay, 2002), with this principle holding true for static (De'sperati, 2003; Laeng \& Teodoresco, 2002; Spivey \& Geng, 2001), movement imagery (Heremans, Helsen, \& Feys 2008), and visual and motor imagery (Poiroux et al., 2015). Thus, eye-movements offer a physical correlate of visual imagery, and provide a useful measure of an individual's imagery engagement (Johansson, et al., 2011). Therefore, here, we replicated Experiment 1, but in addition incorporated eye tracking to provide a more objective measure of imagery use during the tasks. In addition, we also aimed to investigate whether eye movements, physical correlates of visual imagery, differ during varying visual imagery perspective taking. We investigated this by comparing the deviation in eye-movement between a baseline while performing the task and the eye movement during the three conditions of imagery, imagery with sequencing, and math control. Given that eye movements are indicative of the cognitive processes under lying a 
task, we predicted there would be significantly less deviation in eye-movement during IVI than EVI during the imagery condition. Further, due to the possible interference effect for IVI that there would be significantly greater deviation for the IVI group in the imagery and sequencing condition than for the EVI group.

\section{Experiment 2}

\section{Methods}

Participants. Twenty-two different participants (Age $M=21.00, S D=2.66,10$ males) were recruited for this study. As in Experiment 1, participants were screened for imagery ability and handedness, and self-reported as having normal vision. There were 11 participants in each imagery group (IVI or EVI).

Equipment, tasks and procedure. The task and experimental conditions were identical to Experiment 1, with the exception of the imagery scripts, the addition of a baseline trial for each of the target distances, and the number of familiarization trials in each condition. Specifically, due to the demands of the eye tracking equipment, the imagery scripts (in both IVI and EVI groups) were altered to describe only the image of the movement itself. Further, in order to be able to compare eye-movements when actually performing the aiming task (without a condition) and while conducting each of the conditions (Imagery, Imagery with Sequencing, and Math Control) a baseline trial was performed for each of the three respective target distances. Feedback received during a pilot study led to an additional familiarization trial in each condition.

All equipment remained the same as in Experiment 1, apart from the removal of the PLATO Lenses to accommodate the eye tracker. Eye movements were monitored using a non-invasive ASL (model 501, type 2) head mounted eye tracker (Series 5000), with inclusion of a chin rest to ensure that participants head remained in a fixed, stable position throughout the experiment. The eye tracker accurately measured eye line of gaze relative to 
head position, and horizontal displacement of the eye pupil from the fixation cross to the target (accuracy of $0.5^{\circ}-0.1^{\circ}$, sampling at $60 \mathrm{~Hz}$ ). Vertical eye movements were not considered as the task required movement in the horizontal plane only. For each participant in each condition, accuracy of eye movements was assessed by averaging the horizontal eye movements made when actually performing the target distance task in a specific condition and subtracting the horizontal eye movements at baseline for that target distance. This gave a single deviation score for each participant in each condition.

\section{Results}

Participants adhered to their allocated perspective with little variance across conditions (Imagery $M=1.19, S D=0.40$; Sequencing with Imagery, $M=1.00, S D=0.00$ ), and they did not switch from their allocated perspective in either condition (Imagery $M=$ $1.19, S D=0.40 ;$ Sequencing with Imagery, $M=1.29, S D=0.46)$. As before, we checked for differences in imagery ability between groups. An independent samples t-test demonstrated no significant differences in imagery ability between IVI and EVI groups, $t(20)=0.38 p=$ $.71, \beta=.06$.

For task performance (as Experiment 1), we conducted a 2 (Group: IVI, EVI) x 3 (Condition: Imagery, Sequencing with Imagery, Math Control) ANOVA, with repeated measures on Condition. There was no main effect of Group, $F(1,20)=0.78, p=.38$, but a main effect of Condition, $F(2,40)=5.69, p=.007, \eta^{2}=.22, \beta=.84$, replicating the results of Experiment 1 , showing Imagery $(M=75.55)$ had lower errors than Imagery with Sequencing, $(M=87.02)$, and both having lower errors than the Math Control condition $(M=114.65)$. The differences between conditions was again qualified by a significant interaction between Group and Condition, $F(2,40)=3.34, p=.046, \eta^{2}=.14, \beta=.60$. One way ANOVAs for each condition showed a significant decrease in RMSE between the IVI and EVI group in the Sequencing condition, $F(1,20)=4.41, p=.048, \eta^{2}=.18, \beta=1.00$, but no differences in other 
conditions, both $F$ 's $<0.88$. Therefore, as in Experiment 1, the results illustrate that the IVI group had significantly greater error than the EVI group in the Sequencing condition.

Analysis of the eye movement data used the same 2 (Group: IVI, EVI) x 3

(Condition: Imagery, Sequencing with Imagery, Math Control) ANOVA. Similar to the performance data, there was no main effect of Group, $F(1,20)=1.57, p=.22$, but a main effect of Condition, $F(2,40)=13.17, p<.001, \eta^{2}=.39, \beta=.99$, in which, Imagery $(\mathrm{M}=$ 202.90) had less deviation than Imagery with Sequencing, $(M=310.12)$, and both had less deviation than the Math Control condition $(M=391.57)$. Additionally, there was a significant interaction between Group x Condition, $F(2,40)=3.79, p=.031, \eta^{2}=.16, \beta=$ .67 , highlighted in Figure 4. To explore the interaction further, we carried out one-way ANOVAs for each of the conditions. These determined that there was significantly less deviation in the IVI group than the EVI group in the Imagery condition, $F(1,20)=6.19, p=$ $.02, \eta^{2}=.24, \beta=.66$, but no difference in the Imagery with Sequencing and Math Control conditions, both $F \mathrm{~s}<.49, p \mathrm{~s}>.49$.

\section{Discussion}

The results from Experiment 2 replicated Experiment 1 showing that use of visual imagery perspective reduced the average error made by participants in the performance task again supporting research demonstrating the beneficial effects of imagery on learning and human performance (Carrasco \& Cantalapiedra, 2016; Driskell, et al., 1994). Furthermore, for participants using EVI, performance was unaffected by the introduction of an interference sequence dual-task. However, as in Experiment 1, for participants in the IVI group, the sequencing dual-task resulted in poorer performance, supporting our hypothesis that the inclusion of a secondary task that involves the motor system leads to a deterioration of performance when using IVI. In addition, and very interestingly, although our prediction that there would be greater deviation in eye movement in the IVI group in comparison to the EVI 
group in the Imagery with Sequencing condition was not revealed, an interference effect of IVI compared to EVI does appeared in the eye movement results. Specifically, in the imagery condition relative to the EVI condition, IVI showed less deviation in eye movement.. However, this reduced effect was not found when IVI was combined with dual-sequence task, suggesting that IVI was disrupted by the dual-task. There was no evidence of disruptive effects for EVI. Given that eye movements accurately represent the attentive cognitive processes underlying static imagery (De’sperati, 2003; Laeng \& Teodoresco, 2002; Spivey \& Geng, 2001), movement imagery (Heremans et al., 2008), and visual and motor imagery (Poiroux et al., 2015) these results provide evidence that the motor interference task disrupts internal visual imagery, and suggest a reliance of internal visual imagery on dorsal stream visuo-motor neural processes. Furthermore, these finding support the use of eye tracking as a potential measure of an individual's imagery engagement (Johansson, et al., 2011).

\section{General Discussion}

We examined the hypothesis that if different cognitive / neural areas are involved in processing of IVI and EVI, behavioural responses involving imagery should be impacted by a motor dual-task. Across two experiments, we demonstrated that the use of IVI was adversely impacted by the motor dual-task, while the use of EVI was unaffected by the inclusion of the secondary task. The current findings offer support to the hypothesis that internal visual imagery of movement uses similar cognitive processes as those used in actual visual-spatial movement (Decety \& Grezes, 1999). The present research extends this research to specify that IVI seems to be processed within motor cognitive areas, while offering indirect evidence that EVI is less reliant on visuo-motor neural areas (as EVI was unaffected by the dual-task).

Other work has shown that visual imagery of movement has no effect on the activation of the motor network (Stinear, Byblow, Steyvers, Levin, \& Swinnen, 2006). While we do not demonstrate actual activation of the motor network, our findings point to a neural 
dissociation that involves the motor network. There are several explanations for this. Stinear et al. (2006) reported activation of the motor cortex during kinaesthetic imagery, but not visual imagery. The authors attributed these findings to the different organization of the somatosensory to motor cortex compared to the visual to motor cortex. However, the visual imagery perspective was not specified. Based on the results of the present studies, it is possible that the lack of motor network activation was due to the participants using EVI, or a combination of IVI and EVI that washed out the effects. If EVI is processed in the ventral stream, the weaker connections of this stream to motor areas could explain this lack of activation. Moreover, if the authors asked explicitly for the use of an IVI perspective, it is possible there would have been activation of the motor cortex, given the stronger connections. Methodologically, these findings serve to highlight the crucial importance of perspective specificity when investigating visual imagery in research. Indeed, in order to understand imagery cognition, it is important that research delineates visual imagery perspectives.

The findings of the current paper contribute to applied sports and health settings. Researchers and therapists alike advocate the use of imagery as part of rehabilitation for stroke patients (Jackson, Doyon, Richards, \& Malouin, 2004; Lui, Chan, Lee, \& Hui-Chan, 2004). While the theoretical bases for such interventions remain debated (Holmes, 2007), our results indicate that the location of the injury should be taken into account when deciding the imagery perspective to be used by the patient. For example, if the injury occurred in regions of the dorsal stream, the effectiveness of IVI may be attenuated, whereas EVI may bring improvements. Conversely, if the injury was located along the ventral stream, the efficacy of EVI as a treatment perspective might be reduced, whereas IVI may cause improvements. If imagery is to be effectively incorporated into the rehabilitation program of patients with brain 
injury, the interaction between location of damage and imagery perspective might be an important consideration, though clearly more research is needed.

Within the sporting domain, the present findings impact on optimal use of visual imagery. For example, if an individual is using IVI while simultaneously engaging in movement (e.g., a gymnast chalking their hands), the movement may impair the usefulness of the perspective, as it engaged the motor network. These data suggest that concurrent use of EVI with movement will not be affected in the same way, and may result in less interference. From an applied perspective, we could suggest that if imagery were to be performed with movement, EVI could be recommended, as this would place less demand on the athlete's motor-cognitive resources.

While a clear limitation of this study stems from behavioural nature of the study, with inferred cognitive/neural processes, here we provide and discuss several converging strands of evidence that support our hypotheses. Future studies might consider to investigate the effects of IVI and EVI on performance using brain imaging. This research is not easy due to the artefacts that result from performing movements in fMRI, but it could be that the use of eye-tracking during imagery as in Experiment 2 could be used as an alternative task for correlating behavioural and brain measures during the same task.

To conclude, we demonstrate across two experiments that the use of internal visual imagery is disrupted by a motor dual-task, but external visual imagery was unaffected. Using an action dual-task that engages motor networks caused disruption to internal visual imagery processing, supporting previous findings in this area (Deyzac, et al., 2006). These findings provide behavioural evidence that point towards a neural dissociation in for visual imagery perspectives, with the paper providing an essential reference for those conducting visual perspectives imagery research. 


\section{References}

Allbutt, J., Ling, J., Rowley, M., \& Shafiullah, M. (2011). Vividness of visual imagery and social desirable responding: correlations of the vividness of visual imagery questionnaire with the balanced inventory of desirable responding and the Marlowe-Crowne scale. Behavior Research Methods, 43, 791-799. doi:10.3758/s13428-011-0086-8

Beilock, S. L., \& Gonso, S. (2008). Putting in the mind versus putting on the green: Expertise, performance time, and the linking of imagery and action. The Quarterly Journal of Experimental Psychology, 61, 920-932. doi:10.1080/17470210701625626

Burgess, N. (2006). Spatial memory: How egocentric and allocentric combine. Trends in Cognitive Sciences, 10, 551-557. doi:10.1016/j.tics.2006.10.005

Callow, N., \& Hardy, L. (2004). The relationship between the use of kinaesthetic imagery and different visual imagery perspectives. Journal of Sports Sciences, 22, 167-177. doi:10.1080/02640410310001641449

Callow, N., Jiang, D., Roberts, R., \& Edwards, M. G., (2017). Kinesthetic Imagery Provides Additive Benefits to Internal Visual Imagery on Slalom Task Performance. Journal of Sport and Exercise Psychology, 39, 81-86. doi: 10.1123/jsep.2016-0168

Callow, N., \& Roberts, R. (2010). Imagery research: An investigation of three issues. Psychology of Sport and Exercise, 11, 325-329. doi:10.1016/j.psychsport.2010.03.002

Callow, N., Roberts, R., Hardy, L., Jiang, D., \& Edwards, M. G. (2013). Performance improvements from imagery: Evidence that internal visual imagery is superior to external visual imagery for slalom performance. Frontiers in Human Neuroscience, 7, 697. doi:10.3389/fnhum.2013.00697 
Carrasco, G. D., \& Cantalapiedra, A. J. (2016). Effectiveness of motor imagery or mental practice in functional recovery after stroke: a systematic review. Neurologia, 1, 43-52. doi: 10.1016/j.nrl.2013.02.003.

Catalan, M. J., Honda, M., Weeks, R. A., Cohen, L. G., \& Hallett, M. (1998). The functional neuroanatomy of simple and complex sequential finger movements: a PET study. Brain, 121, 253-64. doi:10.1093/brain/121.2.253

Decety, J., \& Grezes, J. (1999). Neural mechanism subserving the perception of human actions. Trends in Cognitive Sciences, 3, 172-178. doi:10.1016/S1364-6613(99)01312-1

De'sperati, C. (2003). Precise oculomotor correlates of visuospatial mental rotation and circular motion imagery. Journal of Cognitive Neuroscience, 15, 1244-59. doi:10.1162/089892903322598184.

Deyzac, E., Logie, R. H., \& Denis, M. (2006). Visuospatial working memory and the processing of visual descriptions. British Journal of Psychology, 97, 217-243. doi: $10.1348 / 000712605 \times 67484$

Di Rienzo, F., Debarnot, U., Daligault, S., Saruco, E., Delpuech, C., Doyon, J., ... Guillot, A. (2016). Online and offline performance gains following motor imagery practice: a comprehensive review of behavioral and neuroimaging studies. Frontiers in Human Neuroscience, 10:315. doi:10.3389\%2Ffnhum.2016.00315

Dragovic, M. (2004). Towards an improved measure of the Edinburgh Handedness Inventory: A one-factor congeneric measurement model using confirmatory factor analysis. Laterality: Asymmetries of Body Brain and Cognition, 9, 411-419. doi:10.1080/13576500342000248

Driskell, J. E., Copper, C., \& Moran, A. (1994). Does mental practice enhance performance? Journal of Applied Psychology, 79, 481-491. doi:10.1037//00219010.79.4.481 
Frank, C., \& Schack, T. (2017). The representation of motor (inter)action, states of action, and learning: Three perspectives on motor learning by way of imagery and execution. Frontiers in Psychology, 8:168. doi: 10.3389/fpsyg.2017.00678

Fourkas, A. D., Ionta, S., \& Aglioti, S. M. (2006). Influence of imagined posture and imagery modality on corticospinal excitability. Behavioural Brain Research, 168, 190-196. doi:10.1016/j.bbr.2005.10.015

Ganesh, S., Cross, E. S., de Lange, F. P., Schie, H. T., \& Wigboldus, D. H. (2015).

Disentangling neural processes of egocentric and allocentric mental spatial transformations using whole-body photos of self and other. NeuroImage, 116, 30-9. doi: 10.1016/j.neuroimage.2015.05.003

Grice, G. R. (1968). Stimulus intensity and response evocation. Psychological Review, 75, 359-73. doi:10.1037/h0026287

Guillot, A., Collet, C., Nguyen, V. A., Malouin, F., Richards, C., \& Doyon, J. (2009). Brain activity during visual versus kinaesthetic imagery: an fMRI study. Human Brain Mapping, 30, 2157-2172. doi:10.1002/hbm.20658

Hall, C. R. (1997). Lew Hardy's third myth: A matter of perspective. Journal of Applied Sport Psychology, 9, 310-313. doi:10.1080/10413209708406490

Hall, C. R., Buckolz, E., \& Fishburne, G. J. (1989). Searching for a relationship between imagery ability and memory of movements. Journal of Human Movement Studies, 17, 89-100.

Hardy, L. (1997). The Coleman Roberts Griffith address: Three myths about applied consultancy work. Journal of Applied Sport Psychology, 9, 277-294. doi:10.1080/10413209708406487 
Hardy, L., \& Callow, N. (1999). Efficacy of external and internal visual imagery perspectives for the enhancement of performance on tasks in which form is important. Journal of Sport and Exercise Psychology, 21, 95-112. doi:10.1123/JSEp.21.2.95

Hebb, D. O. (1968). Concerning imagery. Psychological Review, 75, 466-477. doi:10.1037/h0026771

Heremans, E., Helsen, W. F., \& Feys, P. (2008). The eyes as a mirror of our thoughts: Quantification of motor imagery of goal-directed movements through eye movement registration. Behavioural Brain Research, 187, 351-360. doi:10.1016/j.bbr.2007.09.028

Holmes, P. S. (2007). Theoretical and practical problems for imagery in stroke rehabilitation: An observation solution. Rehabilitation Psychology, 52, 1-10. doi:10.1037/00905550.52 .1 .1

Isaac, A. R. (1992). Mental practice: Does it work in the field? The Sport Psychologist, 6, 192-198. doi: 10.1123/tsp.6.2.192

Jackson, P. L., Doyon, J., Richards, C. L., \& Malouin, F. (2004). The efficacy of combined physical and mental practice in the learning of a foot-sequence task after stroke: A case report. Neurorehabilitation and Neural Repair, 18, 106-111.

doi:10.1177/0888439004265249

Jeannerod, M. (1994). The representing brain: Neural correlates of motor intention and imagery. Behavioural and Brain Sciences, 17, 187-202. doi:

\section{$10.1017 / \mathrm{S} 0140525 \mathrm{X} 00034026$}

Jiang, D., Edwards, M. G., Mullins, P. G, \& Callow, N. (2015). The neural substrates for the different modalities of movement imagery. Brain and Cognition. 97, 22-31. doi: 10.1016/j.bandc.2015.04.005.

Johansson, R., Holsanova, J., \& Holmqvist, K. (2011). The dispersion of eye movements during visual imagery is related to individual differences in spatial imagery ability. In L. 
Carlson, C. Hölscher, \& T. Shipley (Eds.), Proceedings of the 33rd Annual Meeting of the Cognitive Science Society (pp. 1200-1205). Austin, TX: Cognitive Science Society. Kosslyn, S. M. \& Shwartz, S. P. (1977). A simulation of visual imagery. Cognitive Science, 1, 265-295. doi: 10.1207/s15516709cog0103_2

Krigolson, O., \& Heath, M. (2004). Background visual cues and memory-guided reaching. Human Movement Science, 23, 861-877. doi:10.1016/j.humov.2004.10.011

Krigolson, O., Gyn, G. V., Tremblay, L., \& Heath, M. (2006). Is there "feedback" during visual imagery? Evidence from a specificity of practice paradigm. Canadian Journal of Experimental Psychology, 60, 24-32. doi:10.1037/cjep2006004

Laeng, B. \& Teodoresco, D. S. (2002). Eye scanpaths during visual imagery re-enact those of perception of the same visual scene. Cognitive Science, 26, 207-231. doi:10.1207/s15516709cog2602_3

Levine, D. N., Warach, J., \& Farah, M. (1985). Two visual systems in mental imagery: Dissociation of "what" and "where" in imagery disorders due to bilateral posterior cerebral lesions. Neurology, 35, 1010-1018.

Libby, L. K., Valenti, G., Hines, K. A., \& Eibach, R. P. (2014). Using imagery perspective to access two distinct forms of self-knowledge: associative evaluations versus propositional self-beliefs. Journal of Experimental Psychology General, 2, 492-7. doi: $10.1037 / \mathrm{a} 0033705$

Liversedge, S. P., \& Findlay, J. M. (2002). Saccadic eye movements and cognition. Trends in Cognitive Science, 4, 6-14. doi: 10.1016/S1364-6613(99)01418-7.

Lui, K. P., Chan, C. C., Lee, T. M., \& Hui-Chan, C. W. (2004). Mental imagery for promoting relearning for people after stroke: a randomised controlled trial. Archives of Physical Medicine and Rehabilitation, 85, 1403-8. doi:10.1016/j.apmr.2003.12.035 Marks, D.F. (1973). Visual imagery in the recall of pictures. British Journal of Psychology, 
64, 17-24. doi: 10.1111/j.2044-8295.1973.tb01322.x

McCormick, S., Causer, J., \& Holmes, P. (2013). Active vision during action execution, observation and imagery: Evidence for shared motor representations. PLoS ONE, 8, p. e67761. doi:10.1371/journal.pone.0067761Miller, L. E., \& Saygin, A. P. (2013). Individual differences in the perception of biological motion: Links to social cognition and motor imagery. Cognition, 128, 140-148. doi:10.1016/j.cognition.2013.03.013

Moreau, D., Clerc, J., Mansy-Dannay, A., \& Guerrien, A. (2010). Assessing movement imagery ability: Self-report questionnaires vs. performance-based tests. Europe's Journal of Psychology, 4, 93-109. doi:10.5964/ejop.v6i4.225

Norman, J. (2002). Two visual systems and two theories of perception: An attempt to reconcile the constructivist and ecological approaches. Behavioural Brain Sciences, 25, 73-96. doi:10.1017/S0140525X0200002X

Poiroux, E., Cavaro-Ménard, C., Leruez, S., Lemée, J.M., Richard, I., \& Dinomais, M. (2015) What Do Eye Gaze Metrics Tell Us about Motor Imagery? PLoS ONE, 10. doi:10.1371/journal.pone.0143831

Pascual-Leone, A., Dang, N., Cohen, L. G., Brasil-Neto, J. P., Cammarota, A., \& Hallett, M. (1995). Modulation of muscle responses evoked by transcranial magnetic stimulation during the acquisition of new fine motor skills. Journal of Neurophysiolology, 74, 1037-1045.

Poulton, E. C. (1973). Unwanted range effects from using within-subjects experimental designs. Psychological Bulletin, 80, 113-121. doi:10.1037/h0034731

Proteau, L., Tremblay, L., \& Dejaeger, D. (1998). Practice does not diminish the role of visual information in on-line control of a precision walking task: Support for the specificity of practice hypothesis. Journal of Motor Behavior, 30, 143-150. doi:10.1080/00222899809601331 
Roberts, R., Callow, N., Hardy, L., Markland, D., \& Bringer, J. (2008). Movement imagery ability: Development and assessment of a revised version of the vividness of movement imagery questionnaire. Journal of Sport and Exercise Psychology, 30, 200-221. doi:10.1123/jsep.30.2.200

Ross, J. S., Tkach, J., Ruggieri, P. M., Lieber, M., \& Lapresto, E. (2003). The mind's eye: functional MR imaging evaluation of golf motor imagery. American Journal of Neuroradiology, 24, 1036-1044.

Ruby, P., \& Decety, J. (2001). Effect of subjective perspective taking during simulation of action: a PET investigation of agency. Nature Neuroscience, 4, 546-550. doi: $10.1038 / 87510$

Spivey, M., J. \& Geng, J. J. (2001). Oculomotor mechanisms activated by imagery and memory: eye movements to absent objects. Psychological Research, 65, 235-241. doi:10.1007/s004260100059

Stinear, C. M., Byblow, W. D., Steyvers, M., Levin, O., \& Swinnen, S. P. (2006). Kinesthetic, but not visual, motor imagery modulates corticomotor excitability. Experimental Brain Research, 168, 157-164. doi:10.1007/s00221-005-0078-y

Ungerleider, L. G., \& Mishkin, M. (1982). Two visual systems. In D. J. Ingle, M. A. Goodale, \& R. J. W. Mansfield (Eds.), Analysis of Visual Behavior (pp. 549-586). Cambridge, MA: MIT Press.

Vogeley, K., \& Fink, G. (2003). Neural correlates of first-person-perspective. Trends in Cognitive Science, 7, 38-42. doi: 10.1016/S1364-6613(02)00003-7

Waller, D., \& Hodgson, E. (2006). Transient and enduring spatial representations under disorientation and self-rotation. Journal of Experimental Psychology: Learning, Memory and Cognition, 32, 867-882. doi:10.1037/0278-7393.32.4.867 
Witt, S. T., Laird, A. R., \& Meyerand, M. E. (2008). Functional neuroimaging correlates of finger-tapping task variations: an ALE meta-analysis. Neuroimage, 42, 343-56. doi:10.1016/j.neuroimage.2008.04.025

Zhang, H., Long, Z., Ge, R., Xu, L., Jin, Z., Yao, L., Liu, Y. (2014). Motor imagery learning modulates functional connectivity of multiple brain systems in resting state. PLoS ONE, 9. doi:10.1371/journal.pone.0085489 


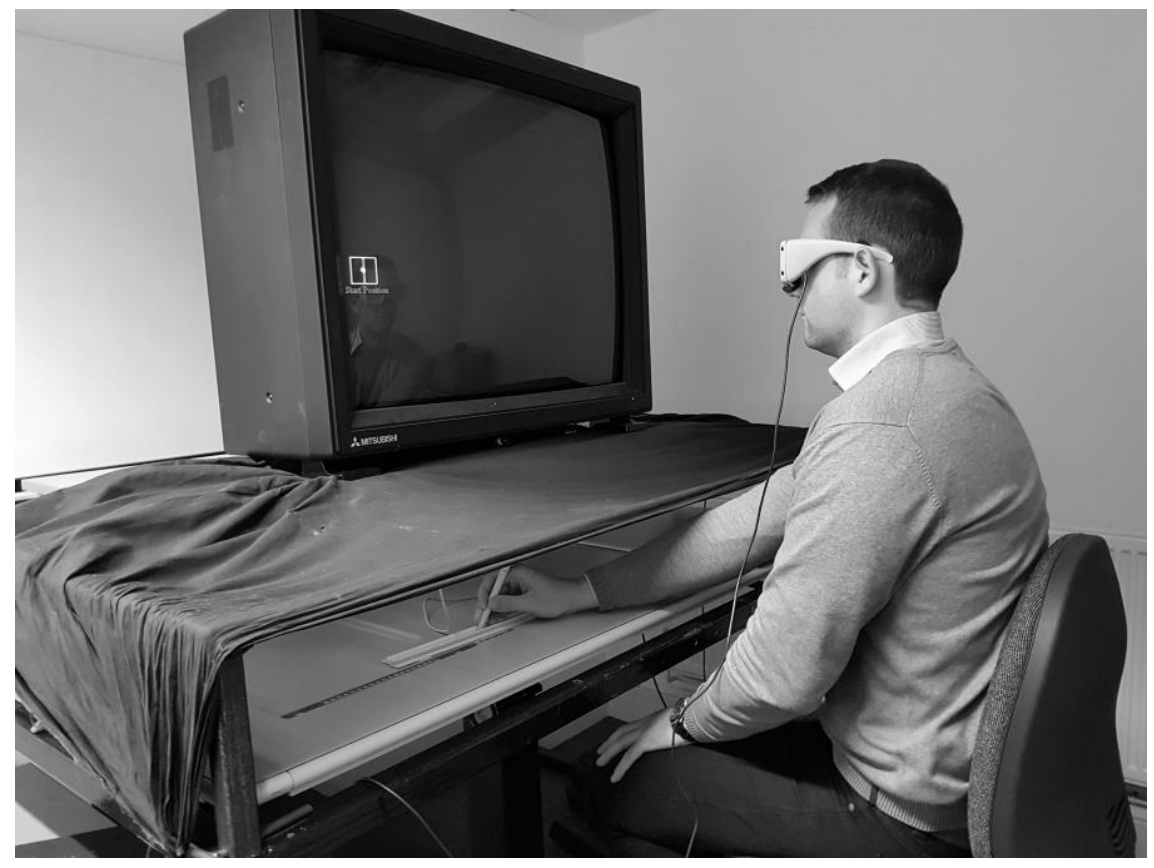

Figure 1. Experiment 1 equipment set-up. 
Start Line

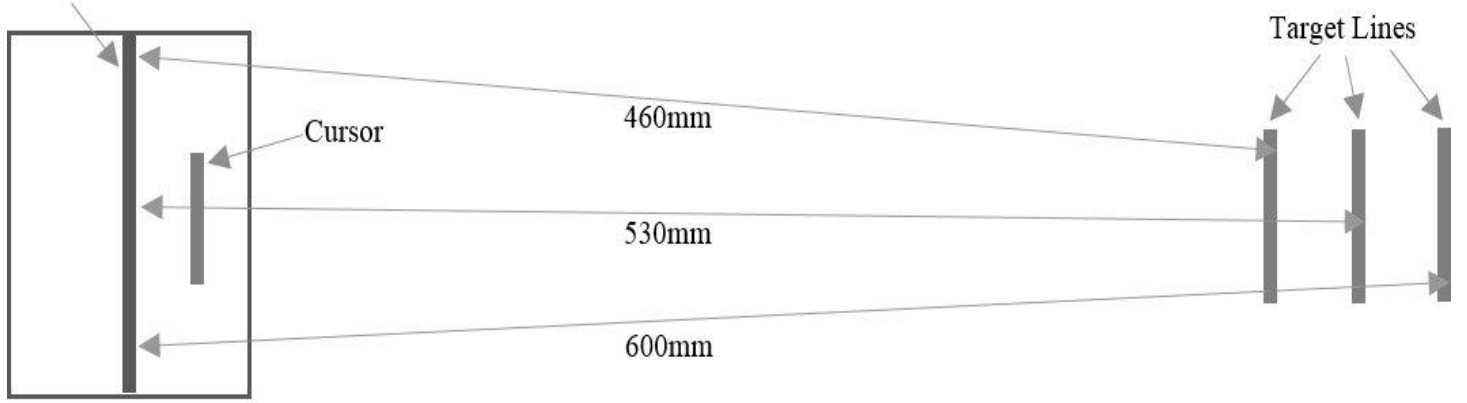

Start Position

Figure 2. Diagrammatical representation of task, note the three target lines were presented individually and randomly across conditions. 


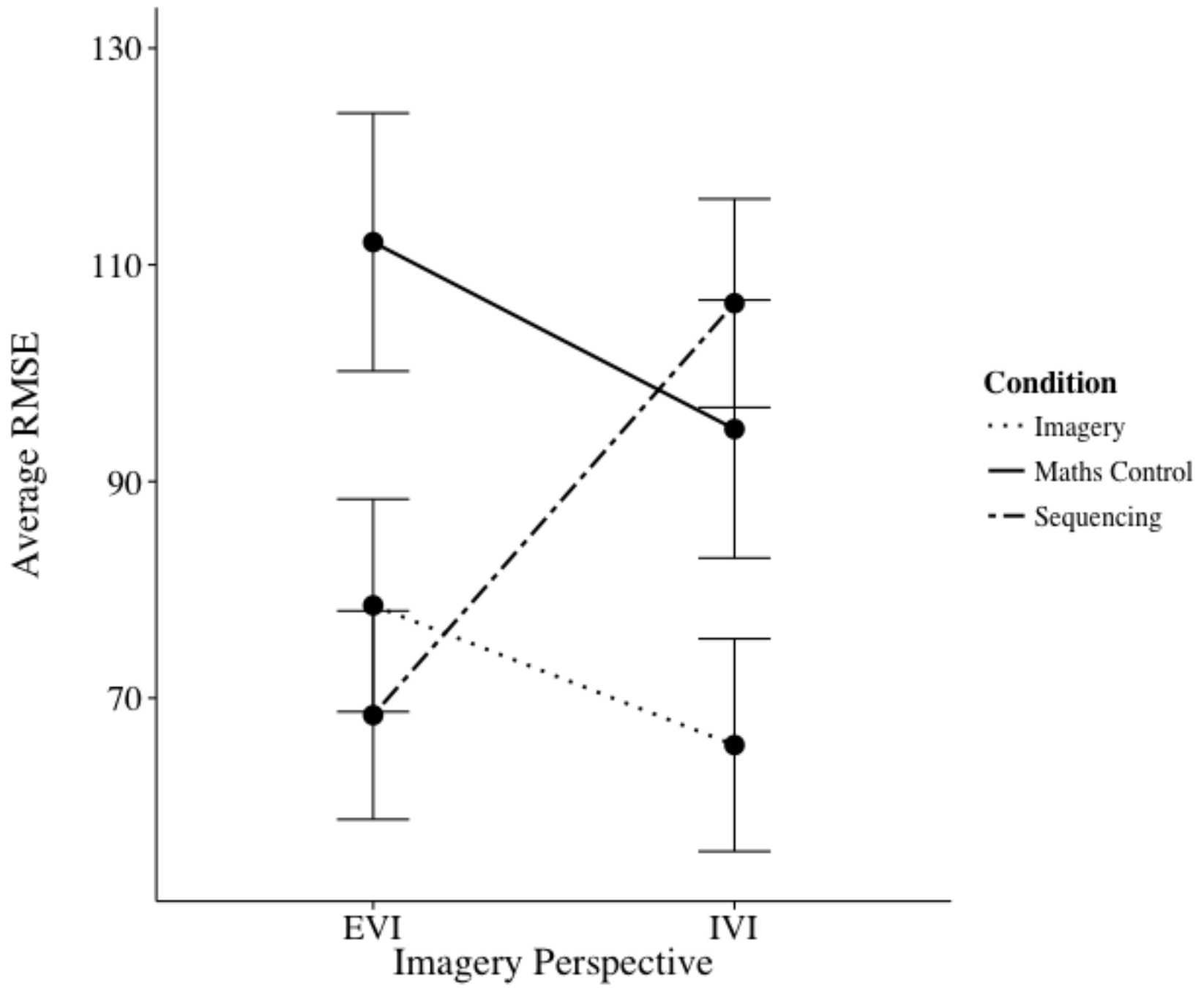

Figure 3. Experiment 1, the average RMSE across imagery perspectives and experimental conditions. Error bars represent \pm 1 SEM. 


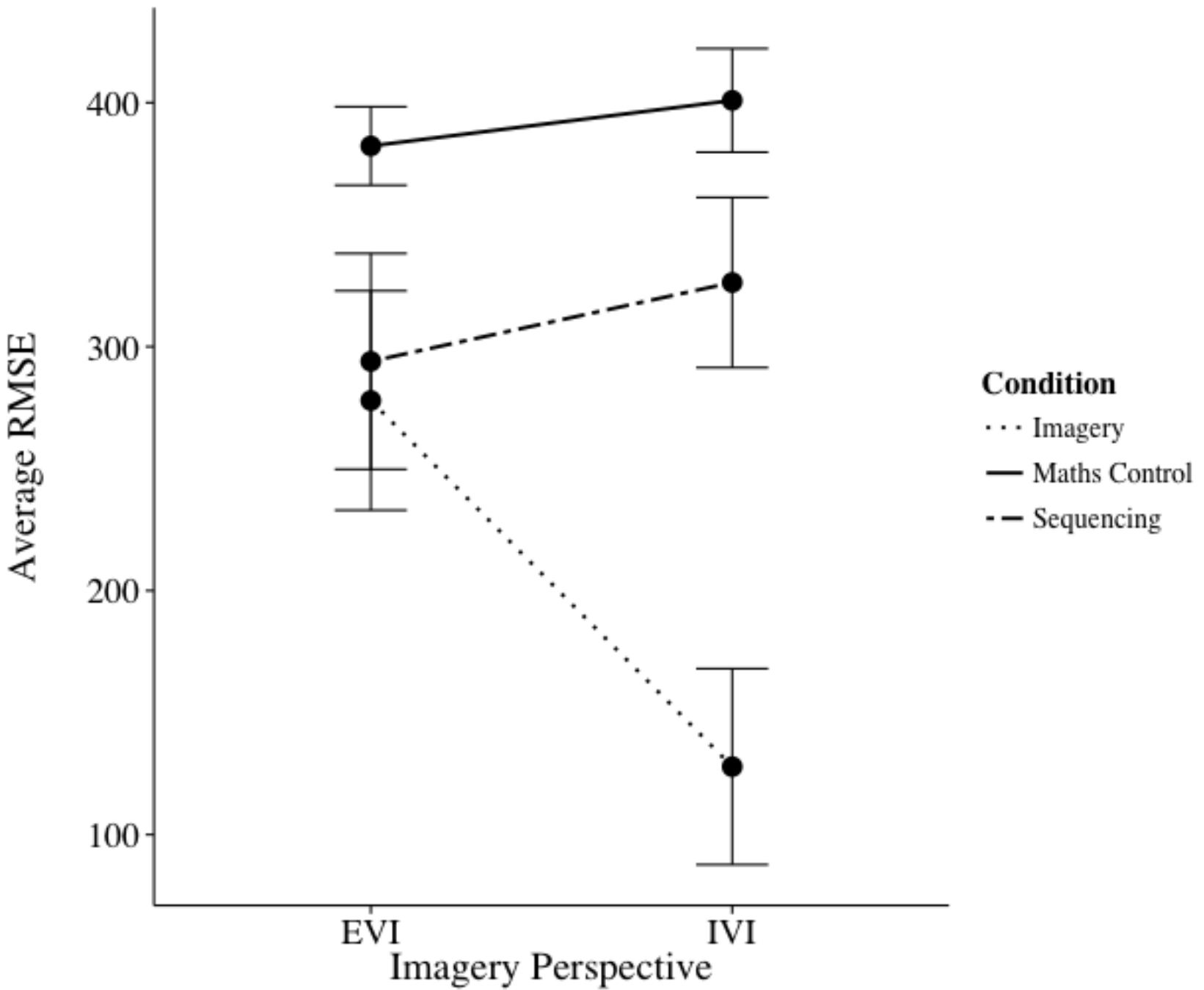

Figure 4. Experiment 2, average eye movement data across imagery perspectives and experimental conditions. Error bars represent \pm 1 SEM. 